IAC-07-A6.3.05

\title{
A SENSITIVITY STUDY OF THE EFFECTIVENESS OF ACTIVE DEBRIS REMOVAL IN LEO
}

\author{
J.-C. Liou \\ ESCG/ERC, Houston, TX USA \\ jer-chyi.liou-1@nasa.gov \\ Nicholas L. Johnson \\ NASA Johnson Space Center, Houston, TX, USA \\ Nicholas.L.Johnson@nasa.gov
}

\begin{abstract}
The near-Earth orbital debris population will continue to increase in the future due to ongoing space activities, on-orbit explosions, and accidental collisions among resident space objects. Commonly adopted mitigation measures, such as limiting postmission orbital lifetimes of satellites to less than 25 years, will slow down the population growth, but will be insufficient to stabilize the environment. Therefore, to better limit the growth of future debris population to protect the environment, the remediation option, i.e., removing existing large and massive objects from orbit, needs to be considered. This paper does not intend to address the technical or economical issues for active debris removal. Rather, the objective is to provide a sensitivity study to quantify the effectiveness of various remediation options. A removal criterion based upon mass and collision probability is developed to rank objects at the beginning of each projection year. This study includes simulations with removal rates ranging from 5 to 20 objects per year, starting in the year 2020. The outcome of each simulation is analyzed and compared with others. The summary of the study serves as a general guideline for future debris removal consideration.
\end{abstract}

\section{INTRODUCTION}

Recent numerical simulations on the evolution of orbital debris populations in low-Earth orbit (LEO, 200 to $2000 \mathrm{~km}$ altitude) indicate that the population has reached a point where the environment is unstable and population growth is inevitable ${ }^{1-2}$. The main conclusion from the two studies is that even if no further space launches were conducted, the Earth satellite population would remain relatively constant for only the next 50 years or so. Beyond that, the debris population would begin to increase noticeably due to the production of collisional debris. In reality, the satellite population growth in LEO will undoubtedly be worse than the studies indicate, since spacecraft and their orbital stages will continue to be launched into space and unexpected major breakups may continue to occur. Postmission disposal of vehicles, such as limiting postmission orbital lifetimes to less than 25 years, can certainly slow down the population growth $^{3-5}$. However, this mitigation measure will be insufficient to prevent further growth of the Earth satellite population. To better preserve the near-Earth environment for future space activities, other alternatives must be considered.

Concepts for removing large debris from LEO have been proposed for more than 25 years. Early ideas for using the U.S. Space Shuttle, either directly or in conjunction with an orbital transfer vehicle, were found unattractive due to safety, availability, cost, and policy issues. Numerous independent robotic concepts, ranging from classical space-based garbage scows to momentum and electrodynamic tethers, drag augmentation devices, solar and magnetic sails, and other exotic techniques, have also been considered. However, reviews by panels of international experts have repeatedly failed to identify a single plan which is both technically 
feasible in the near-term and economically viable.

Nonetheless, in late 2006 the International Academy of Astronautics (IAA) initiated a new study to determine if a nexus of technology, cost, and policy might lead to an achievable means of remediating the near-Earth space environment in the foreseeable future. Although the IAA study will not be completed until late 2008 or 2009, the purpose of the present paper is to describe the potential effectiveness of debris removal operations under various scenarios. These results, in turn, could influence the development of efficient debris removal techniques.

This paper summarizes a sensitivity study on the effectiveness of active debris removal (ADR). A non-mitigation scenario where the historical LEO population was simulated, then projected 200 years into the future, was adopted as the benchmark scenario. A simple selection criterion based on collision probability and mass of each $10 \mathrm{~cm}$ and larger object was developed. Numerical simulations of three ADR scenarios with different removal rates were carried out and compared with the benchmark population. The results demonstrated that with a reasonable selection criterion active debris removal could be a very effective way to limit the growth of future debris populations.

\section{MODEL DESCRIPTION}

The tool used in the study is the NASA orbital debris evolutionary model LEGEND (a LEO-toGEO Environment Debris model). It is a high fidelity, three-dimensional, physical model. LEGEND is capable of simulating the historical and future debris environment from LEO to the geosynchronous orbit (GEO) regions (Liou et al. 2004; Liou 2007). The focus of the present study is the LEO environment. Each test scenario presented included 100 Monte Carlo simulations with a projection period of 200 years. Future launch traffic was simulated by repeating the 1999-to-2006 launch cycle. The solar flux F10.7 values used in the projection period had two components: a short-term projection (2006-2007, obtained from NOAA Space Environment Center) and a long-term projection (2008-2206).
The long-term F10.7 projection ${ }^{8}$ was a repeat of a sixth-order sine and cosine functional fit to Solar Cycles 18 to 23. A simple smoothing function was used to interpolate the two solar flux components during the short- to long-term transition. Explosion probabilities of rocket bodies and spacecraft were based on an analysis of historical explosions between 1988 and 1998. Objects with non-zero explosion probabilities were classified, by origin and type, into nine categories. Each category was assigned a timedependent explosion probability for up to 10 years (since launch). Only objects $10 \mathrm{~cm}$ and larger were included in collision consideration. This is also the same size threshold for objects to be discussed in the following analysis. After each breakup, fragments were generated with the NASA Standard Breakup Model, which described the size, area-to-mass ratio, and velocity distributions of the debris ${ }^{9}$.

A key component of LEGEND is its collision probability estimation module. Collision probabilities among orbiting objects are estimated with a fast pair-wise comparison algorithm, Cube ${ }^{7,10}$. Since objects are evaluated individually, it becomes possible to develop selection criteria for ADR studies. For any object $i$ that has a finite collision probability with a second object $j$, within the same cube at time $t$, its collision probability can be expressed as:

$$
d P_{i}(t)=s_{i} s_{j} V_{i m p} \sigma d U d t
$$

where $s_{i}$ and $s_{j}$ are the spatial densities of objects $i$ and $j$ in the cube, respectively, $V_{i m p}$ is the relative velocity between the two objects, $\sigma$ is the combined collision cross-sectional area, $d U$ is the volume of the cube, and $d t$ is the time interval. The default parameters used in LEGEND include a time step of 5 days, and $10 \mathrm{~km} \times 10 \mathrm{~km} \times 10$ km cubes.

The first step in an ADR study is to develop a set of selection criteria so that objects selected for removal will have the most effect in reducing the long-term growth of the LEO debris population. Objects with perigee altitudes above $2000 \mathrm{~km}$, or with eccentricities greater than 0.5 , were not considered for removal in this study. Payloads 
launched after 2006 were assumed to have 8-year mission lifetimes, and were not considered for removal until after the end of the mission. For any object $i$ which was eligible for removal consideration, a simple criterion, $R_{i}$, was adopted:

$$
R_{i}(t)=P_{i}(t) \times m_{i},
$$

where $P_{i}(t)$ was defined by Equation (1), and $m_{i}$ was the object's mass. Debris removal was implemented at the beginning of each projection year after the year 2020. Once the $R_{i}$ values for objects with non-zero collision probability were calculated, they were sorted in descending order. A pre-defined number of objects with the highest $R_{i} \mathrm{~S}$ were removed from the simulated environment immediately.

\section{ACTIVE DEBRIS REMOVAL CASE STUDY}

Four test scenarios were selected for this case study. The first one was a non-mitigation ("business-as-usual") scenario. The other three scenarios assumed ADR was implemented in the year 2020, with annual debris removal rates of 5, 10 , and 20 objects, respectively (these three scenarios were referred to as 2020/5, 2020/10, and 2020/20 throughout the rest of the paper). Each scenario was carried out with a 200 -year projection period and $100 \mathrm{MC}$ runs.

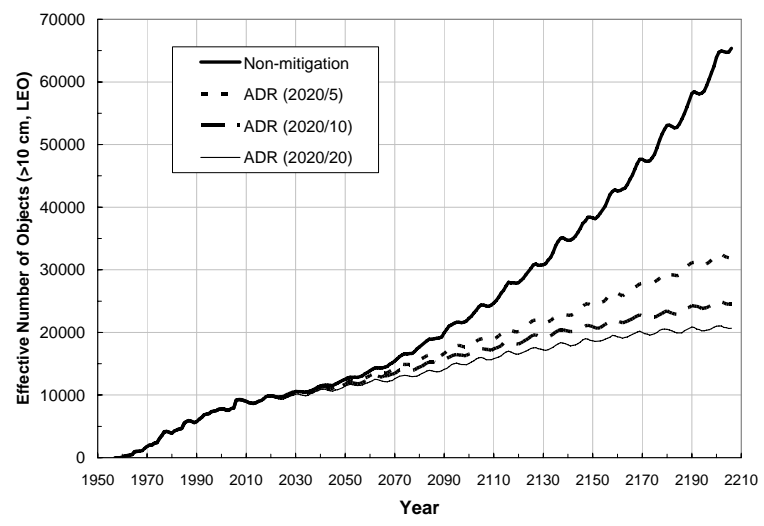

Figure 1: LEGEND-simulated LEO debris populations (objects $10 \mathrm{~cm}$ and larger) between 1957 and 2006 (historical), and between 2007 and
2206 (future projection). Each curve represented the average of 100 Monte Carlo runs.

Figure 1 shows the effective numbers of objects, $10 \mathrm{~cm}$ and larger, in LEO from the four scenarios. The effective number is defined as the fractional time, per orbital period, an object spends between 200 and $2000 \mathrm{~km}$ altitude. Each curve represented the average of 100 Monte Carlo runs. The business-as-usual scenario predicted a fast non-linear growth of the future LEO debris population in the next 200 years. The three ADR scenarios, on the other hand, predicted a much slower increase in the environment. A simple parameter, Effective Reduction Factor (ERF), can be calculated to quantify the effectiveness of each ADR scenario using the non-mitigation scenario as a benchmark. ERF is defined as:

ERF $=$ [total no. of objects reduced in 2206] / [no. of objects removed via ADR through 2206].

The ERF for the ADR 2020/5 scenario is 36. It means for every object that is removed (via ADR) from the environment, a total of 36 objects will be reduced by the end of 2206 . Table 1 summarizes the ERFs of the three ADR scenarios. The overall result indicates that Equation (1) is indeed a good objective selection criterion to identify objects which have the greatest potential of contributing to the growth of the future debris population. The trend also shows that, as expected, not all objects contribute equally to future collision-fragmentation activities. Once the worst potential contributors are removed, the effectiveness of ADR will be reduced. 


\begin{tabular}{cccc}
\hline & ADR 2020/5 & ADR 2020/10 & ADR 2020/20 \\
\hline $\begin{array}{c}\text { NUMBER OF OBJECTS REMOVED VIA ADR } \\
\text { THROUGH } \\
2206(A)\end{array}$ & 935 & $\mathbf{1 8 7 0}$ & $\mathbf{3 7 4 0}$ \\
$\begin{array}{c}\text { REDUCTION IN LEO OBJECTS IN 2206 (B) } \\
\text { ERF BY 2206 = (B) / (A) }\end{array}$ & 33,337 & $\mathbf{4 0 , 9 0 7}$ & $\mathbf{4 4 , 7 1 2}$ \\
\hline
\end{tabular}

Table 1.: The ERFs of the three active debris removal scenarios. The LEO population (objects $10 \mathrm{~cm}$ and larger) from the non-mitigation scenario is used as the benchmark for the comparison.

Another way to measure the effectiveness of ADR is to examine the total collision probability of the LEO population as a function of time (Figure 2). The cumulative collision probabilities, by 2006, for the non-mitigation, ADR 2020/5, ADR 2020/10, and ADR 2020/20 scenarios are 172.9, 74.2, 55.4, and 45.8, respectively. They are summarized in Table 2 . The last row of the table is the ratio of objects removed, via ADR, to the reduction in cumulative collision probability by 2206 . In the 2020/5 scenario, for every 9.5 objects removed via ADR, one collision is reduced by 2206. This number increases with an increasing removal rate. Again, the trend is a reflection of how ADR is implemented in the study - the worst potential offenders are removed first from the environment.

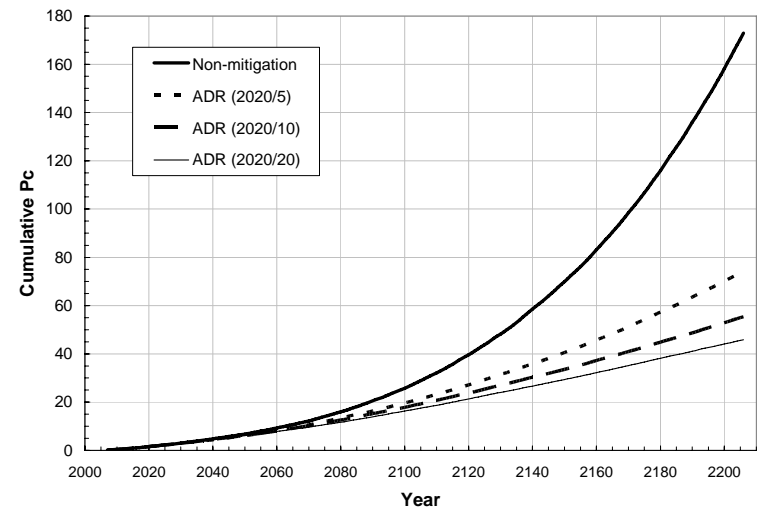

Figure 2: Cumulative collision probabilities as functions of time from the four test scenarios. Each curve is an average of $100 \mathrm{MC}$ runs.
The spatial density distributions of the $10 \mathrm{~cm}$ and larger LEO objects are shown in Figure 3. The bottom curve is the environment at the end of 2006. The other four curves, from top to bottom, are the predicted environment in 2206 from the non-mitigation, ADR 2020/5, ADR 2020/10, and ADR 2020/20 scenarios, respectively.

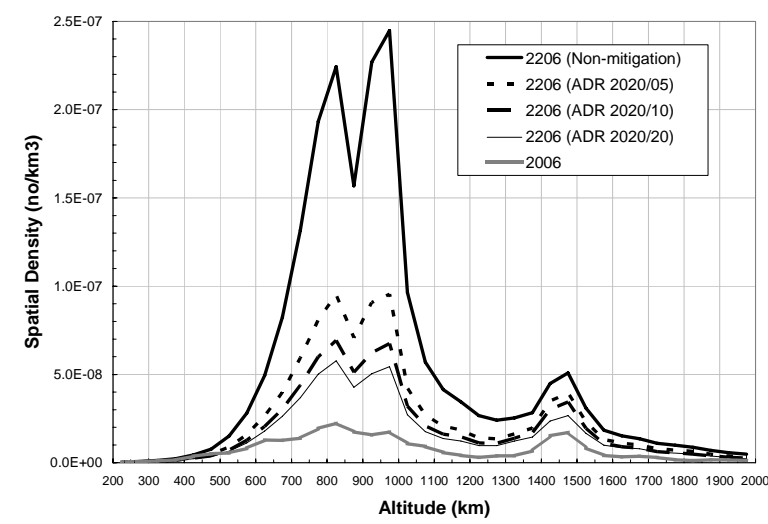

Figure 3. Spatial density distribution of objects $10 \mathrm{~cm}$ and larger in LEO. The bottom curve represents the LEO environment at the end of 2006. The other four curves, from top to bottom, are the predicted environment in 2206 from the nonmitigation, ADR 2020/5, ADR 2020/10, and ADR 2020/20 scenarios.

The population reduction in high collision activity regions (between 800 and $1000 \mathrm{~km}$ ) due to active debris removal is very effective. This is also a good testament to validate the selection criterion of Equation (1). Figures 4 to 6 are the distributions in inclination, altitude, and mass of objects removed from the three ADR scenarios. The altitude of each object is where its collision probability was evaluated for ADR selection per Equation (1). 


\begin{tabular}{cccc}
\hline & ADR 2020/5 & ADR 2020/10 & ADR 2020/20 \\
\hline $\begin{array}{c}\text { NUMBER OF OBJECTS REMOVED VIA } \\
\text { ADR THROUGH } \\
\text { 2206 (A) }\end{array}$ & 935 & $\mathbf{1 8 7 0}$ & 3740 \\
$\begin{array}{c}\text { REDUCTION IN CUMULATIVE COLLISION } \\
\text { PROBABILITY BY 2206 (C) }\end{array}$ & $\mathbf{9 8 . 7}$ & $\mathbf{1 1 7 . 6}$ & $\mathbf{1 2 7 . 1}$ \\
(A) / (C) & $\mathbf{9 . 5}$ & $\mathbf{1 5 . 9}$ & $\mathbf{2 9 . 4}$ \\
\hline
\end{tabular}

Table 2: Effectiveness of active debris removal in terms of cumulative collision probability of the environment. The LEO population (objects $10 \mathrm{~cm}$ and larger) from the non-mitigation scenario is used as the benchmark for the comparison.

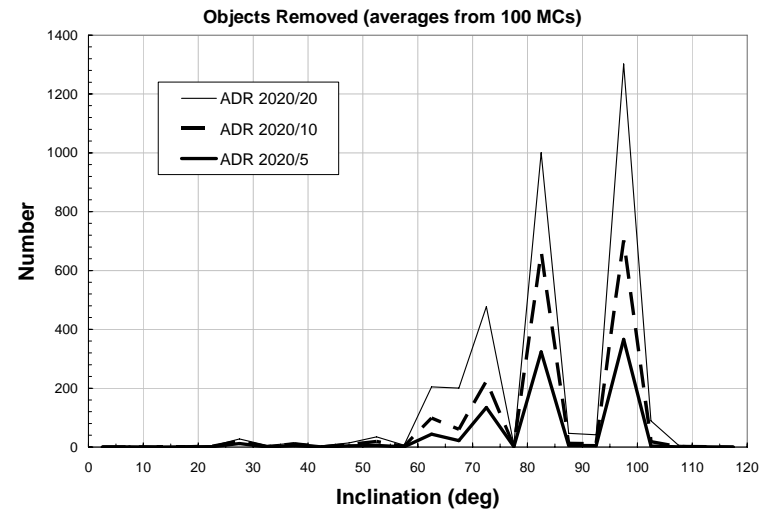

Figure 4. Inclination distributions of objects removed from the three ADR scenarios. Each distribution is the average of 100 Monte Carlo runs.

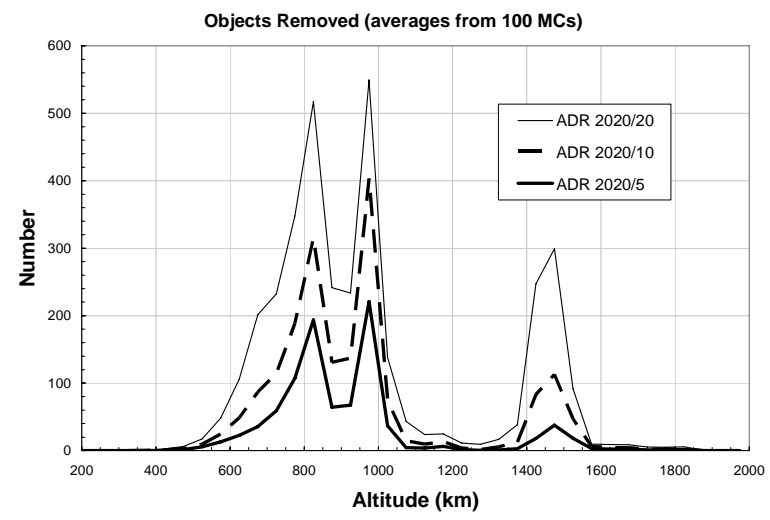

Figure 5. Altitude distributions of objects removed from the three ADR scenarios. Each distribution is the average of 100 Monte Carlo runs.

These three distributions paint a clear picture of objects with the greatest potential of contributing to future LEO collision fragmentations. They are (1) massive objects between 1000 and $1500 \mathrm{~kg}$ and between 2500 and $3000 \mathrm{~kg}$, (2) objects with inclinations in one of the three bands: $70^{\circ}-75^{\circ}$, $80^{\circ}-85^{\circ}$, and $95^{\circ}-100^{\circ}$, and (3) objects that spend a significant amount of time in one of the three altitude regions: $800 \mathrm{~km}-850 \mathrm{~km}, 950 \mathrm{~km}-1000$ $\mathrm{km}$, and $1450 \mathrm{~km}-1500 \mathrm{~km}$.

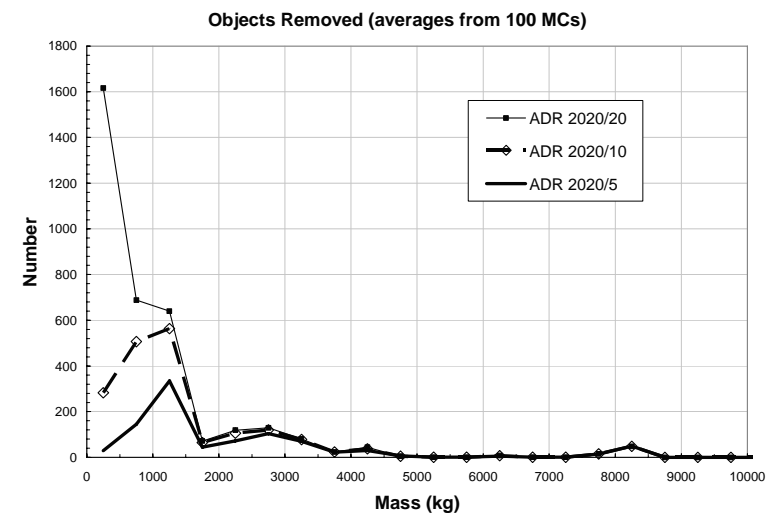

Figure 6. Mass distributions of objects removed from the three ADR scenarios. Each distribution is the average of 100 Monte Carlo runs.

Figure 7 presents a different way to examine the growth of the future debris population. The top four cures are the masses in LEO as functions of time from the four test cases. The total mass in LEO is calculated based on the mass of each LEO-crossing object weighted by the fraction of time the object resides in LEO. Although the breakup of a massive object adds a significant amount of debris to the environment, and increases the impact risks to other orbiting satellites, it does not lead to any increase in mass in LEO. For the non-mitigation scenario, there is a constant increase in mass as a result of the imbalance between objects being launched and objects naturally decaying. When ADR is implemented, significant amounts of mass are removed (the bottom three curves), and total mass in LEO is kept more or less constant through 2206. The mass factor will need to be carefully evaluated when a cost/benefit analysis 
is required for the actual implementation of active debris removal.

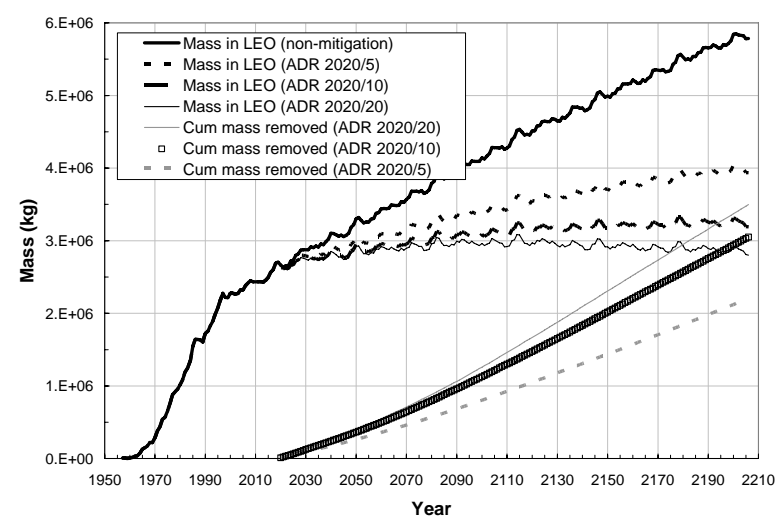

Figure 7. Top four curves: masses in LEO from the four test scenarios. Each LEO-crossing object's mass is weighted by its time residing between 200 and $2000 \mathrm{~km}$ altitudes. Bottom three curves: cumulative masses of objects removed from the three ADR scenarios.

\section{DISCUSSION}

The test cases described in the previous section represented the first step to evaluate the effectiveness of ADR. The scenarios were designed to demonstrate and quantify, in a relative way, how ADR could reduce the growth of the future debris population in LEO. The assumptions made in the simulations were reasonable, but certainly not perfect. The nonmitigation scenario was selected as a benchmark for comparison. Since mitigation measures (such as limiting postmission orbital lifetimes of satellites to less than 25 years) have been implemented, although not $100 \%$ by major space agencies, the assumption of "non-mitigation" might be too conservative. Other scenarios, including the "no new launches" and "postmission disposal", for benchmarks to test the effectiveness of various ADR strategies. Quantitatively the results would be different. It is expected that fewer objects would be required to be removed per year to achieve the same reduction factor. Qualitatively, however, ADR scenarios would still be an effective way to control the population growth. Another assumption in the ADR simulations was that once an object was selected, it was removed from the environment immediately. This is obviously the best case scenario. Many removal techniques currently being suggested would only shorten the orbital lifetime of the target object. The impact of this will reduce the ERFs listed in Table 1 somewhat.

The objects identified to have the greatest potential of contributing to future population growth through 2206 (Figures 4-6) depend on two factors. First, objects in the current environment. Second, objects included in the repeated 1999-to-2006 launch cycle through 2206. Spacecraft and upper stages launched in the future will certainly be different from those in the assumed launch cycle. The true distributions in inclination, altitude, and mass of the potentially worse offenders will be different from those in Figures 4 to 6 . However, the selection criterion of Equation (1) will always be able to identify the objects that need to be considered for removal first.

One possible modification to improve Equation (1) is to include the longevity factor. When two objects have similar " $R$ " values, it is obvious that removing the object which has a longer orbital lifetime would have a more positive impact on the environment. The difficulty here is how to properly incorporate the lifetime dependence into the equation. Since orbital lifetime does not increase linearly with increasing altitude, a linear dependence of orbital lifetime to " $R$ " may result in the removal of all objects at higher altitudes, e.g., $1500 \mathrm{~km}$, before any objects at lower altitudes are considered. The present study addressed the near-term (i.e., next 200 years or so) environment concerns. ADR scenarios based on the selection criterion of Equation (1) effectively reduce the population in regions which have the greatest potential of growth in 200 years (see Figure 3). If the objective is to address the population growth over a much loner period of time ${ }^{11}$, e.g., thousands of years, the direct implementation of orbital lifetime into the selection criterion may be further justified.

The year selected to implement active debris removal in the three ADR scenarios was 2020. It was assumed that by then the technical and cost challenges would be addressed to make ADR a reality. Moving the starting time somewhat 
further into the future (while the population growth is still linear) should only postpone the population reduction accordingly. However, if ADR is not implemented before the population reaches a much faster or even exponential growth rate, the cost-to-benefit ratio of ADR would be significantly increased.

The actual implementation of any mitigation measures to remove objects from space is very complicated. The cost and technical challenges are the two major obstacles that will not be resolved in the near future. Additional issues, such as ownership, liability, and policy need to be addressed as well. However, if the mitigation measures currently adopted by international space agencies and industry are insufficient to limit the growth of the future debris population, active debris removal must be seriously considered as an option to preserve the nearEarth space for future generations.

\section{REFERENCES}

1. Liou, J.-C. and Johnson, N.L. Risks in space from orbiting debris, Science, 311, 340-341, 2006.

2. Liou, J.-C. and Johnson, N.L. Instability of the present LEO satellite populations, Adv. Space Res., doi:10.1016/j.asr.2007.04.081, in press, 2007.

3. Krisko, P.H., Johnson, N.L., Opiela, J.N. EVOLVE 4.0 orbital debris mitigation studies, Adv. Space Res., 28, 9, 1385-1390, 2001.

4. Walker, R., Martin, C.E., Stokes, P.H., Wilkinson, J.E., Klinkrad, H. Analysis of the effectiveness of space debris mitigation measures using the DELTA model, Adv. Space Res., 28, 9, 1437-1445, 2001.

5. Liou, J.-C., Johnson, N.L. A LEO Satellite postmission disposal study using LEGEND, Acta Astronautica, 57, 324-329, 2005.

6. Liou, J.-C., D. T. Hall, P. H. Krisko, and J. N. Opiela., LEGEND - A three-dimensional LEO-to-GEO debris evolutionary model. Adv. Space Res. 34, 5, 981-986, 2004.
7. Liou, J.-C., Collision activities in the future orbital debris environment, Adv. Space Res., 38, 9, 2102-2106, 2006.

8. Whitlock, D. Modeling the effect of high solar activity on the orbital debris environment, Orbital Debris Quarterly News, 10, 2, pp. 4, NASA Johnson Space Center, Houston, Texas, 2006

9. Johnson, N. L., P. H. Krisko, Liou, J.-C., and P. D. Anz-Meador, NASA's new breakup model of EVOLVE 4.0, Adv. Space Res., 28, 9, 1377-1384, 2001.

10. Liou, J.-C., D. J. Kessler, M. J Matney, and E. G. Stansbery, A new approach to evaluate collision probabilities among asteroids, comets, and Kuiper Belt objects. Proc. Lunar Planet. Sci. Conf. 34, 1828, 2003.

11. Kessler, D. J. and Anz-Meador, P. D. Critical number of spacecraft in low Earth orbit: Using satellite fragmentation data to evaluate the stability of the orbital debris environment, in Proceedings of the 3rd European Conference on Space Debris, ESA SP-473, 265-272, 2001. 